\title{
U-CLX Model Proposal Using the Standard XAPI
}

\author{
Mauricio Ramirez \\ Universidad de Cauca, FIET, IDIS \\ Universidad Nacional Abierta y a \\ Distancia \\ Popayán, Colombia \\ gmr@unicauca.edu.co
}

\author{
Cesar Collazos \\ Universidad de Cauca, FIET, IDIS \\ Popayán, Colombia \\ ccollazo@unicauca.edu.co
}

\author{
Fernando Moreira \\ REMIT, IJP, Universidade \\ Portucalense, Porto \\ IEETA, Universidade de Aveiro, \\ Aveiro \\ Portugal \\ fmoreira@upt.pt
}

\begin{abstract}
The Ubiquitous Learning model, based on learning experiences and connective learning for higher education U-CLX, is a proposal to generate a new dimension of U-Learning. The model explores the possibility of implementing the XAPI standard to manage the learning experiences that are generated in a ubiquitous learning process, the model has two components, a pedagogical component and a technological component.
\end{abstract}

Keywords - U-Learning; Connective Learning; Experience Learning; XAPI Standard; Model.

\section{INTRODUCTIÓN}

The evolution of information and communication technologies (ICT) applied to education have allowed the development of education in different environments, where technology has greatly permeated the way to develop current educational processes. Nowadays, carrying out educational processes and specifically learning processes without the technology support is practically impossible, the digitalization of society forces and generates the need in education to implement new ways of teaching and learning.

The roles of educational actors have changed, currently students or apprentices have become the main actors in educational processes, teachers in advisors or companions of students in their educational processes and technology has become the most important means to develop education [1].

The trends of education and current learning processes are not developed by compulsory or pre-established curricula, they are developed from the students' learning needs, the learning processes are defined according to the interests of the students. In accordance with this way, nowadays we should to start talking about lifelong learning, meaningful learning, etc., but with a new term called learning experiences LE, these learning experiences are the group and union of the different learning forms. The needs and interests of students for their life and their professional future lead to a education personal processes and specific. These groupings and unions of personal learning processes, specific needs, specific interests, forms of learning, etc., are called learning experiences.

From these changes in the roles of students, teachers have also modified their work; today a teacher is a cognitive mediator or tutor of the students' learning experiences and according to this also becomes a designer of learning experiences that will help and contribute them to achieve their learning processes.

Teachers and students make use of ICT as a mean to develop learning experiences, since these offer the necessary tools to carry out educational processes and learning processes. Without technology it would be practically impossible to carry out personalized learning experiences and teachers could not provide accompaniments to these processes and experiences, which is why information and communication technologies are necessary in the development of current education.

The way and the reason of learning are in constant change, the needs of society, science, humanity and technologies demand new ways of doing different activities, also leaving aside jobs and professions that have traditionally been offered today, new skills and knowledge for acquiring and learning appear [2].

One of the most important concepts in current education is ubiquity [2], the development of educational and learning processes at any time, place and in any medium has generated the possibility of providing new ways of carrying out educational processes, learning processes and in general it has revolutionized education opening new possibilities to develop new research and studies in which ubiquity is the fundamental factor.

In general, the possibility of carrying out ubiquitous educational processes or U-Learning is one of the important research areas because it allows the combination of different technologies and components of education to improve educational processes with the aim of improving education in general. In this moment does not exist a u-learning model that combine connective learning and experience learning xAPI standard.

The paper aims to present the proposal of a U-Learning model, in which a pedagogical component and a technological component are implemented. A detailed description is made of the developments carried out so far. The pedagogical component is based on the use of constructivist theory and specifically on connective learning [3] and on the technological 
component side, it is based on the use of the standard of learning experiences or XAPI [4]. The combination of these components generates a new dimension of U-Learning and contributes to the development of technology applied to education, specifically ubiquitous virtual higher education.

In the case of the Ubiquitous Learning model based on connective learning and learning experiences in English, Ubiquitous Learning support by Connective Learn and Learning (U-CLX) aims to define a learning experience involving technological elements such as a system of learning management that supports the standard, a gamification engine, a social learning tool and a native tool that uses the standard natively [2].

The definition of a U-Learning ecosystem is proposed in which the aforementioned technologies are included, in this ecosystem a learning experience will be defined within a specific context, in which the student or apprentice interacts in a formal and informal way, such a way that within the learning experience a flow of learning is generated with the use of the different technologies proposed.

This paper is structured in the following way, in the first point the introduction to the topic is presented, in the second point the body of the document is described, where the conceptualization of the model is framed, the U- Learning and the XAPI standard, in the third point the proposal of the U-CLX model is presented, with the work done, the fourth point presents the validations of the model, the fifth point presents the conclusions and future work and to finish the acknowledgments and references used in the document.

\section{BACKGROUND}

\section{A. U-Learning}

U-Learning or ubiquitous learning is - in general terms - the learning process that can be developed at any time, place, context and device [3] [4]. For other authors, U-Learning is the evolution of E-Learning and M-Learning, generating a new learning model [5]. The characteristics of U-Learning pose challenges in the pedagogical and technological part.

The term ubiquity suggests learning as omnipresent, that is, as a process developed and distributed over time, in space and in different contexts. In the ubiquitous learning is learned in a conscious or unconscious, making use of different means of learning such as: 1) e-learning; 2) mobile or m-learning, 3) learning games or g-learning, 4) simulators, 5) virtual worlds, 6) serious games, 7) activities in the real world, 8) experimental learning, 9) social learning, 10) learning not online, 11) collaborative learning, 12) learning in the inverted classroom, among others. Additionally, it can be added that ubiquitous learning is the union of formal and informal learning [6] [7] [8].

From the perspective of U-Learning, the learning process is done formally and informally, the learning process can be developed at any time and place, context and in any way, suggesting that with current technological tools it is not possible to measure the ubiquitous formal and non-formal learning processes, that is, it is not possible to measure learning experiences and/or those activities carried out in the context of ubiquity [10].

In this sense, the possibility of defining the Ubiquitous Learning or ubiquitous learning as an ecosystem of learning processes and technologies focused on education is proposed, this proposed ecosystem for U-Learning is the union of different definitions of U-Learning proposals by the authors who have worked on the development of the concept [11].

According to this, the U-Learning ecosystem is considered as the technological medium in which different technological components that share the same habitat or medium come together, in this case the technological and communication media. That is, in U-Learning converge e-learning, m-learning, g-learning, t-learning, v-learning, b-learning, among others, which coexist independently and related to each other and in different combinations, which allow the development of ULearning in different moments, spaces, contexts and means, the union of information and communication technologies focused on education allow to generate the omnipresence of ULearning.

Figure 1 shows a cloud of facts with the convergence of the different technologies that comprise the U-Learning ecosystem.

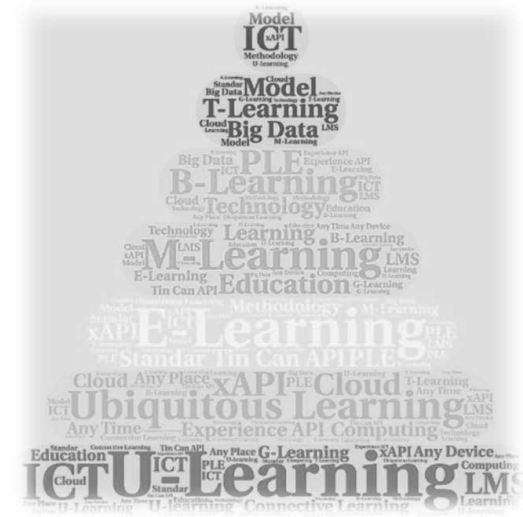

Figure 1. U-Learning Ecosystem (author source).

The concept of Ubiquitous Learning or U-Learning is approached from the development of omnipresent educational processes that can be developed at any time, place, context, device, medium, through any technology of information and communication, both formally as informal, to summarize the ubiquitous learning process makes use of all the technologies that are at your fingertips to develop learning processes. According to this, the U-CLX model presented below makes use of the concept previously proposed for U-Learning [12].

The model presented below is focused on the management of learning experiences and connective learning for higher education U-CLX to develop a new dimension of U-Learning.

B. $x A P I$

The technological component of the model has the technological support of ubiquitous computing, pervasive computing and the standard of learning experiences or xAPI or Tin Can API or Experience API (abbreviations referring to the 
same standard) [13] [14]. The xAPI is an evolution and improvement of the SCORM standard.

The standard of learning experiences xAPI is a new specification for learning technologies, the standard allows to collect data in different technological elements, media and spaces whether on or off line. This is done through a structure or format that defines the standard in order to manage the data and generate information regarding the different learning experiences that a person can have. The standard also defines the vocabulary that should be used, the way to communicate, capture and share the information between the different systems that relate to the standard or make use of the standard.

The data of the learning experiences that are captured, are stored in a Learning Record Store LRS and from the LRS the data is obtained to perform the learning analysis process or Learning Analytics, with this information you can know the learning experiences that a person can have with any way or technology, the learning process, the characterization of learning, the type and manner of learning, the profile of learning and in this way better decisions can be made to improve the educational, teaching and learning processes.

The xAPI standard, defines the functions and how to capture the data in a specific way and in a predefined format, which allows obtaining the information of different technologies, software, contents, devices, among others, with which the students or apprentices interact. Also, with the standard it is possible to generate learning flows or interaction with different technological elements that develop learning experiences. An experience can start with a software or with a content in a specific context and end in another space, with another technology generating a learning flow in which a learning experience is developed, in this process, it is in which the standard gets involved. Information throughout the learning flow and/or the entire learning experience [15].

The learning experiences are recorded and stored in an LRS (Learning Record Store), this storage is made according to a previously defined structure of the following form person-verbobject-complement. In this structure, the form and how to save the data of the learning experiences is defined. In addition, a TDS (Training Delivery System), which is a system that allows the delivery and monitoring of learning content and learning experience in the technological elements will be used with which we want to work [16].

Once these elements are defined, the standard begins with the process of analyzing the learning experiences or in English LA (Learning Analytics) with which the whole process of knowledge of the learning experiences obtained from the data will be carried out. Obtained with the LRS and that allow to draw conclusions and future work in the improvement of the learning processes.

The standard can be applied with any technology that is currently, it can be a technology that works over the internet or without internet, it can also relate different technologies to each other, or it can relate different technologies through an LRS or relate several LRS to each other and manage the information in such a way that it allows to store information in a single storage or in different storage, all this is done according to the structure given by the standard and the way it is defined to store and manage the data.

The xAPI standard is not very well known at the moment, although it has been developed and published for several years, this has meant that the use and application is developing slowly and its applications in technologies and products are not very broad.

The possibilities of developing ubiquitous learning processes with the XAPI standard opens up the possibility to develop new combinations of information and communication technologies, in such a way that U-Learning can be developed naturally taking into account the definition of the ecosystem of U-Learning raised in this paper and thus include formal and informal learning, learning at any time, place, context, medium and device, combining the concepts of different authors and defining the technological and pedagogical ecosystem of learning ubiquitous or U-Learning [17].

The XAPI standard allows managing ubiquitous learning or U-Learning, that is, the development of learning in different contexts; with different elements and technologies; that is why it defines the way to collect data from learning experiences to store, share and manage [19].

\section{PROPOSAL OF THE U-CLX}

The Ubiquitous Learning model, based on learning experiences and connective learning for higher education UCLX, is a proposal to generate a new dimension of U-Learning that explores the possibility of implementing the XAPI standard to manage the learning experiences that are generated in a ubiquitous learning process, the U-CLX model has two components, a pedagogical component and a technological component.

The pedagogical component is based on the theory of constructivism specifically in an evolution of this theory. Connective learning [18] is a form of evolution of this theory, it raises the form of learning through learning networks. According to this, connective learning applies the concepts of networks in learning processes, where the concepts of nodes and relationships between the nodes are presented.

According to the above, the node refers to any object, person, etc. The relationships between the nodes is the learning process. It is important to be clear that the main node in this learning network is the student or the learner. In this sense it can be said that each person has its own learning network and that between people multiple connections can be made between their networks and this generates different learning processes due to their multiple relationships [19].

Due to the conceptualization of what U-Learning is and the learning process, in the pedagogical component of the U-CLX model other concepts are also worked on, such as collaborative learning, online learning, active methodologies, the invested classroom and collaboration, for a learning process to be ubiquitous, the formal and informal educational process must be taken into account. 
The technological component of the model has the technological support of ubiquitous computing, pervasive computing and the standard of learning experiences or xAPI.

The XAPI standard, is a standard that allows collecting and managing the data of learning experiences that are developed in different moments, spaces, contexts, media, technologies and other elements in which learning processes can be developed that can be measured, saved and manage, to generate knowledge regarding learning experiences. In this context, a learning experience is any activity, event or set of activities and/or events that allows new knowledge to the student or the apprentice [20].

The model proposes the possibility of including combinations of different pedagogical strategies and technological elements; however, for the development of the practical case for tests, some elements of the pedagogical component and elements in the technological component will be selected and, in this way, to test and validate the model with data to make of the learning experiences of the students in a specific academic context. In this sense, the development of learning experiences in a context of virtual higher education and specifically in the development of learning and collaborative work

Figure 2 depicts an initial view of the U-CLX. Model.

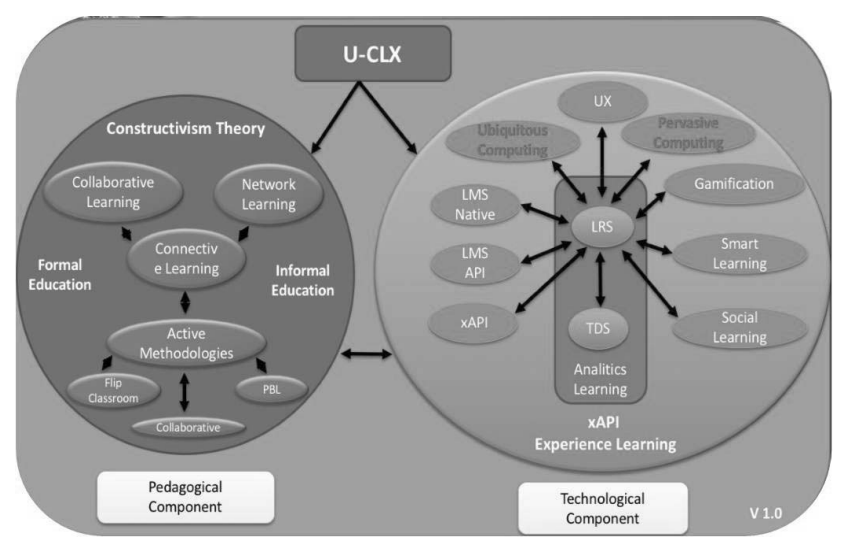

Figure 2. U-CLX model (author source).

The previous figure presents the initial vision of the U-CLX model, where the two pedagogical and technological components are defined, in the pedagogical component the use of collaborative learning, active methodologies, online learning and formal and informal learning, and in the technological component raised the use of ubiquitous computing, pervasive computing, elements of gamification, xAPI standard, smart devices among others.

In the pedagogical component is located the constructivist theory that is the fundamental basis of the model, specifically with connective learning which is an evolution or improvement since it presents elements that allow to explain the current educational and learning processes through the technologies of the information and communication, there is also collaborative learning and network learning, formal and informal learning, learning activity methodologies such as flip classroom and problem-based learning, all these elements framed in the development of the U- Learning.

In the technological component, ubiquitous computing and the XAPI standard are presented as the fundamental basis of the model, pervasive computing, user experience, learning management systems or LMS related to the xAPI standard, gamification, intelligent learning and social learning and the analysis of learning as a base element in the management of the technological component.

The aforementioned elements are those that were initially proposed to be developed in the model, before starting with the validation processes by experts in the field where the U-CLX model was presented, the operation and relationships were explained in a face-to-face interview and then a questionnaire was delivered about the model, in this questionnaire questions were asked about the viability of the model and the existing relationships, in the end a general and specific feedback was delivered by the experts. Six expert evaluations were carried out and a new version of the model was proposed with the answers to the questionnaires, feedbacks and comments.

The model has also been presented at different international events, specifically in doctoral symposia where observations and comments have been made that have nurtured the development of it [5] [21] [22].

With the expert's validations made to the model in different academic spaces, personal and congresses presentations, interviews and doctoral symposiums, the first conclusion was reached, the U-CLX model was being considered in a broad and general way, since it contained many pedagogical and technological elements, in accordance with these validations and with new revisions of the model generated a version with fewer elements.

Figure 2 shows an update view of the U-CLX. Model.

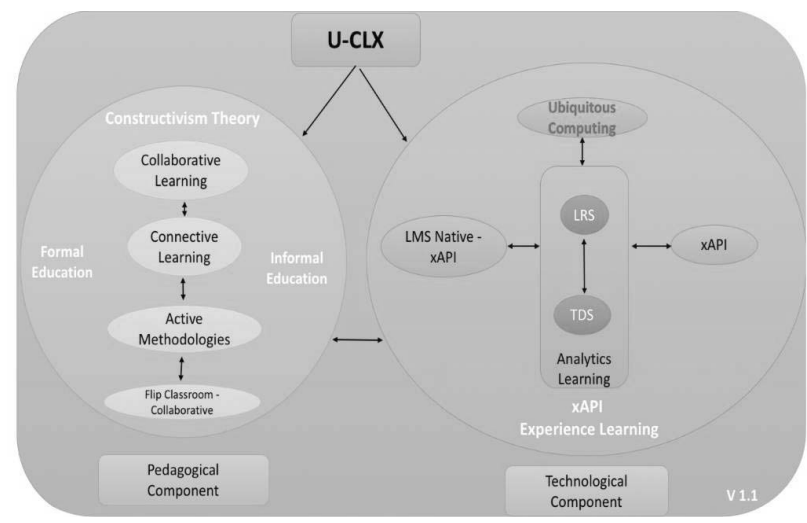

Figure 3. U-CLX model update version (author source).

Figure 3 presents the update version of the U-CLX model, in this version is where the two pedagogical and technological components are defined. In the pedagogical component the use of collaborative learning, and formal and informal learning is 
considered, and in the technological component it is raised the use of ubiquitous computing and the XAPI standard.

The new version of the model is in a new validation process with experts, to start the new design and development process of the U-CLX model, the idea with the model is to carry out a new validation process with interviews, presentations and the compatibility of the technology to verify the possibility to develop the model and technological component with the standard. In this way to start with the application of the model making use of the indicated technologies under the support of the pedagogical component. The definition of the elements of the learning experiences under the xAPI standard and the processes of management and validation of the data with the proposal model are set.

\section{VALIDATIONS}

The model has been initially implemented through configurations and functional tests, the use of the Moodle and the specification of the xAPI standard has been defined to connect the LMS with the standard, in accordance with this an initial structure of the learning experience has been developed according to the standard to relate it to the LMS and generate the initial data to store and manage with the learning analysis system.

The following tests are presented with data focused on collaborative learning, according to this the structures of the learning experiences must be defined, connect the LMS with the data of the collaborative works and carry out the learning analysis process and thus obtain information about collaborative learning in terms of performance, learning processes, profiles and types of learning.

This learning experience will capture the data of each of the technological tools through the standard, Figure 3 presents the updated version of the U-CLX model, in this version where the two pedagogical and technological components are defined, in the pedagogical component the use of collaborative learning, and formal and informal learning is proposed, and in the technological component the use of ubiquitous computing and the xAPI standard was proposed. [23].

The new version is in a new validation process with experts and to start the design, development and validation process of the U-CLX model, the idea with the model is to carry out a new validation process with experts in the subject and verify if it is possible to develop the model, in this way start with the application of the model making use of the indicated technologies under the support of the pedagogical component. According to this, the definition of the elements of the learning experiences under the xAPI standard is defined and the processes of management and validation of the data with the model are set.

The model has been initially implemented through configurations and functional tests, the use of the Moodle and the specification of the XAPI standard has been defined to connect the LMS with the standard, in accordance with this an initial structure of the learning experience has been developed. According to the standard to relate it to the LMS and generate the initial data to store and manage with the learning analysis system. The following tests are presented with data focused on collaborative learning, according to this the structures of the learning experiences must be defined, connect the LMS with the data of the collaborative works and carry out the learning analysis process and thus obtain information about collaborative learning in terms of performance, learning processes, profiles and types of learning. In this learning experience the data of each of the technological tools through the XAPI standard will be captured, making use of the LRS and the TDS to track and manage the learning contents. Accordingly, the structure for capturing the data for each of the tools must be defined and the flow of learning in the defined context must be continued [23].

The U-CLX model will again be put to consideration and validation with experts in its new version, and also the configurations and data and information obtained initially with the tests carried out.

The final concept of the U-CLX model is a ubiquitous learning model that integrates a pedagogical component based on constructivist theory, specifically connective learning, relating formal and informal ubiquitous collaborative learning and a technological component based on ubiquitous computing that is based on the use of the standard of learning experiences that stores, manages and analyzes the experiences of a learning management platform.

\section{CONCLUSIONS AND FUTURE WORK}

The implementation of a ubiquitous model in which a technological ecosystem is defined that includes different technologies and that complies with the characteristics of ubiquity, poses a major challenge in the technological area, for this model it was necessary to define the concept of ULearning and to start this conceptualization it was necessary to consider the technological development and the inclusion of technologies, especially the xAPI standard, which is the technological basis for the development of the U-CLX Model.

The definition of U-Learning was proposed as an ecosystem in which different conceptualizations of authors who have worked on this issue converge, merge and combine, and in the same line, the mixture of different information and communication technologies applied to educational processes and of learning.

The change of roles in current education is also considered, especially with the inclusion of the concept of ubiquity in education and the development of U-Learning, as well as the possibilities of developing new areas and dimensions of education. The U-CLX model and its parts were presented, as well as the development and application process that has been carried out and is planned to be carried out with the new validations and the inclusion of new elements that allow carrying out the application and validation of the model.

The possibility of developing the model allows generating a new dimension and a new space for the application of ubiquitous technologies in education, the implementation of the standard of learning experiences or xAPI in the data management processes and the generation of information from learning experiences open the possibility to develop educational processes at any time, place and context in a personalized way, which will help teachers to have better 
information to support students in their learning interests and students to know better the development of its educational and academic processes.

Regarding future work, the validation of the model is proposed through the inclusion of other technological tools that allow the evaluation of more data within the given context, the inclusion of multiple learning contexts and, in general, the inclusion of new elements that allow for the realization of the evaluation of the U-CLX model in different learning processes, contexts, media, times, spaces. What will allow to nourish and evaluate the model so that it can reach a state of maturity. To perform the validation of the U-CLX model, the application and development of the model with the technologies indicated therein, to perform the functional and conceptual tests, define the application elements of the model and the data that will be used to manage the learning of the models to the student's learning experiences in collaborative work. It raises the need to perform new functional tests with the Moodle LMS, the structures of collaborative learning experiences in a ubiquitous way, the learning analysis system with a more robust testing environment that allows to manage more data and obtain more collaborative learning information, performance, learning processes, profiles and types of learning, which will allow a better development of the model in terms of technology.

We must continue working on the development and application of the xAPI standard in the context of the U-CLX model in such a way as to improve the operation of the model and perform a better management of the learning for the case study that is proposed to test and validate the model. Once the data of the U-CLX ecosystem is obtained, the process of information management will be carried out through a learning analysis process, in which the information and knowledge will be obtained to evaluate if the learning experience is successful. or it is not successful in the context of the collaborative learning experience defined to test the model and improve it. In the future work, it is proposed to make a prototype of the model in which the structure of the defined learning experiences is stored in the LRS and a learning analytical process can be performed to manage the learning experiences in a ubiquitous way from the technological perspective.

The model proposes to manage ubiquitous learning through the definition of students' learning experiences in any context, making use of any technology, which can be developed at any time and place, all in order to store and manage the learning experiences of the students and that allows to feed information to the different educational actors involved in these processes, to make better and more accurate decisions.

\section{REFERENCES}

[1] M. Zapata-Ros, "Teorías y modelos sobre e 1 aprendizaje en entornos conectados y ubicuos: Bases para un nuevo modelo teorico a partir de una visión crítica del 'conectivismo,"” Teor. la Educ., vol. 16, no. 1, pp. 69-102, 2015.

[2] G. M. Ramirez, C. A. Collazos, F. Moreira, and C. González, "Relation Between U-learning, Connective Learning, and Standard xAPI: A
Systematic Review," in Proceedings of the XVIII International Conference on Human Computer Interaction, 2017, p. 3:1--3:4.

[3] M. C. Tumino and U. Adventista, "Conectivismo: Hacia El Nuevo Paradigma De La Enseñanza Por Competencias," Eur. Sci. J., vol. 12, no. 10, pp. 112-129, 2016.

[4] M. Manso-Vazquez, M. Caeiro-Rodriguez, and M. Llamas-Nistal, "XAPI-SRL: Uses of an application profile for self-regulated learning based on the analysis of learning strategies," 2015 IEEE Front. Educ. Conf., pp. 1-8, 2015.

[5] R. Software, "Tin Can API." [Online]. Available: https://tincanapi.com/tin-can-xapi/.

[6] M. Weiser, "The Computer for the 21st Century," Sci. Am., vol. 265, no. 3, pp. 94-104, 1991

[7] C. Collazos, J. Jurado, and L. Merchan, Entornos Ubicuos y Colaborativos (U-CSCL) para Ambientes de Enseñanza-Aprendizaje de Competencias Profesionales. Cali, 2016.

[8] M. Coto, C. a. Collazos, and S. Mora-Rivera, "Modelo Colaborativo y Ubicuo para apoyar los procesos de enseñanza-aprendizaje a nivel Iberoamericano,” Rev. Educ. a Distancia, no. 48, 2016.

[9] E. Fernandez, U-Learning El Futuro esta aqui. Mexico, 2010.

[10] B. Gros, Kinshuk, and M. Maina, The Future of Ubiquitous Learning: Learning Desings for Emerging Pedagogies. Lecture Notes in Educational Technology. Springer International Publishing, 2016.

[11] A. Flores, "Reflexiones en torno al aprendizaje ubicuo, desde la visión CTS," pp. 177-200, 2012.

[12] C. S. González, C. A. Collazos, and R. García, "Desafío en el diseño de MOOCs: incorporación de aspectos para la colaboración y la gamificación,” RED. Rev. Educ. a Distancia, vol. 7, no. 48, pp. 1-23, 2016.

[13] Siemens, "Connectivism: A Learning Theory for the Digital Age," 2004.

[14] H.-J. Jung, "Ubiquitous Learning: Determinants Impacting Learners' Satisfaction and Performance With Smartphones," Lang. Learn. Technol., vol. 18, no. 183, pp. 97-119, 2014.

[15] B. H. Kang and H. Kim, "Proposal: A design of u-learning module application for multi-cultural students in Korea," Int. J. Softw. Eng. its Appl., vol. 9, no. 1, pp. 167-172, 2015.

[16] J. M. Kevan and P. R. Ryan, "Experience API: Flexible, Decentralized and Activity-Centric Data Collection," Technol. Knowl. Learn., vol. 21, no. 1, 2016.

[17] J. M. Kevan and P. R. Ryan, "Experience API: Flexible, Decentralized and Activity-Centric Data Collection," Technol. Knowl. Learn., vol. 21, no. 1, 2016.

[18] J. Yahya,Ahmad, "The definition and characteristics of ubiquiteous learning," Int. J. Educ. Dev. Using Inf. Commun. Technol., vol. 6, no. 1, pp. 117-127, 2010

[19] R. Software, "Tin Can API." [Online]. Available: https://tincanapi.com/tin-can-xapi/.

[20] E. A. Amrieh, T. Hamtini, and I. Aljarah, "Preprocessing and analyzing educational data set using X-API for improving student's performance," 2015 IEEE Jordan Conf. Appl. Electr. Eng. Comput. Technol., pp. 1-5, 2015.

[21] G. Siemens, S. Onderwijsdagen, D. Age, E. Design, G. Siemens, S. Downes, G. Siemens, and D. Age, "Connectivism: a new learning theory ?," 2006.

[22] G. M. Ramirez, C. A. Collazos, and F. Moreira, "A Systematic Mapping Review of All-Learning Model of Integration of Educational Methodologies in the ICT," in Recent Advances in Information Systems and Technologies: Volume 2, Á. Rocha, A. M. Correia, H. Adeli, L. P. Reis, and S. Costanzo, Eds. Cham: Springer International Publishing, 2017, pp. 897-907.

[23] G. M. Ramirez, C. A. Collazos, and F. Moreira, "All-Learning: The state of the art of the models and the methodologies educational with ICT," Telemat. Informatics, Oct. 2017. 